\title{
THE STRENGTH OF THE RAINBOW RAMSEY THEOREM
}

\author{
BARBARA F. CSIMA AND JOSEPH R. MILETI
}

January 14, 2009

\begin{abstract}
The Rainbow Ramsey Theorem is essentially an "anti-Ramsey" theorem which states that certain types of colorings must be injective on a large subset (rather than constant on a large subset). Surprisingly, this version follows easily from Ramsey's Theorem, even in the weak system $\mathrm{RCA}_{0}$ of reverse mathematics. We answer the question of the converse implication for pairs, showing that the Rainbow Ramsey Theorem for pairs is in fact strictly weaker than Ramsey's Theorem for pairs over $R C A_{0}$. The separation involves techniques from the theory of randomness by showing that every 2-random bounds an $\omega$-model of the Rainbow Ramsey Theorem for pairs. These results also provide as a corollary a new proof of Martin's theorem that the hyperimmune degrees have measure one.
\end{abstract}

\section{INTRODUCTION}

We begin by recalling Ramsey's Theorem for the set $\omega$ of natural numbers.

Definition 1.1. Given a set $Z$ and $n \in \omega$, we let $[Z]^{n}=\{x \subseteq Z:|x|=n\}$.

Definition 1.2. Suppose that $n, k \in \omega$ and $f:[\omega]^{n} \rightarrow k$. We say that a set $H \subseteq \omega$ is homogeneous for $f$ if $H$ is infinite and $f$ is constant on $[H]^{n}$.

Theorem 1.3 (Ramsey's Theorem). Let $n, k \in \omega$ and suppose that $f:[\omega]^{n} \rightarrow k$. There exists a set $H$ homogeneous for $f$.

The effective content and reverse mathematical strength of Ramsey's Theorem have been extensively studied (see Jockusch [9]; Seetapun, Slaman [15]; and Cholak, Jockusch, Slaman

B. Csima was partially supported by Canadian NSERC Discovery Grant 312501. 
[3] for many of the central results). In particular, Ramsey's Theorem for pairs (that is, restricting to the case $n=2$ ) has emerged as an interesting case study in reverse mathematics because it is not equivalent to any of the "big five" systems to which most previously studied theorems were equivalent (see Simpson [16] for an introduction to Reverse Mathematics). This behavior of Ramsey's Theorem for pairs arises because diagonalization techniques show that homogeneous sets for computable colorings need not be simple, while [15] and [3] show how little can be coded into such sets.

Hirschfeldt and Shore [8] considered many combinatorial statements which are simple consequences of Ramsey's Theorem for pairs such as "Every infinite linear ordering has either an infinite ascending or infinite descending sequence" and "Every infinite partial ordering has either an infinite chain or an infinite antichain". They showed that these and other statements are in fact strictly weaker and built an intricate web of implications/nonimplications in this realm. Many of their techniques for showing that a specific statement is strictly weaker than Ramsey's Theorem for pairs involve either showing that computable instances of the statement always have low solutions or in showing one can find solutions to computable instances which fail to compute any diagonally noncomputable function. Below, we establish a new technique using measure-theoretic considerations.

The key restriction in Ramsey's Theorem is that the number of colors used is finite. In contrast, the Rainbow Ramsey Theorem restricts attention to colorings considered where each individual color can be used only some fixed finite number of times.

Definition 1.4. Let $n, k \in \omega$. A function $f:[\omega]^{n} \rightarrow \omega$ is $k$-bounded if $\left|f^{-1}(c)\right| \leq k$ for every $c \in \omega$.

Definition 1.5. Suppose that $n \in \omega$ and $f:[\omega]^{n} \rightarrow \omega$. We say that a set $R \subseteq \omega$ is a rainbow for $f$ if $R$ is infinite and $f$ is injective on $[R]^{n}$. 
Theorem 1.6 (Rainbow Ramsey Theorem). Let $n, k \in \omega$ and suppose that $f:[\omega]^{n} \rightarrow \omega$ is $k$-bounded. There exists a set $R$ which is a rainbow for $f$.

The Rainbow Ramsey Theorem (and Ramsey's Theorem itself) both follow easily from the much more general Canonical Ramsey Theorem of Erdös and Rado (see Mileti [13] for an effective analysis of this theorem). However, Galvin noticed that the Rainbow Ramsey Theorem follows easily from Ramsey's Theorem.

Proof of the Rainbow Ramsey Theorem. Fix a well-ordering $\prec$ of $[\omega]^{n}$. Define $g:[\omega]^{n} \rightarrow k$ by letting

$$
g(x)=\mid\left\{y \in[\omega]^{n}: y \prec x \text { and } f(y)=f(x)\right\} \mid
$$

By Ramsey's Theorem, there exists a set $H$ homogeneous for $g$. Suppose that $x, y \in[H]^{n}$ with $x \neq y$. Since $g(x)=g(y)$ and either $x \prec y$ or $y \prec x$, it follows by definition of $g$ that $f(x) \neq f(y)$. Therefore, $H$ is a rainbow for $f$.

The natural question now is whether one can recover Ramsey's Theorem from the Rainbow Ramsey Theorem in a similarly simple manner. In other words, are these statements simply "duals" of each other? Todorcevic [18] and Abraham, Cummings, and Smyth [1] investigated these questions in the uncountable realm. In particular, they showed that the question of whether the Rainbow Ramsey Theorem for 2-bounded colorings of pairs holds at $\aleph_{1}$ is independent of $Z F C$ (whereas Ramsey's Theorem for colorings of pairs with 2 colors provably fails at $\aleph_{1}$ ). Also, in the finite realm, the Rainbow numbers for pairs grow polynomially (see Alspach, Gerson, Hahn, Hell [2] and Hell, Montellano-Ballesteros [7]) while the Ramsey numbers grow exponentially. We show below that when working on $\omega$, the Rainbow Ramsey Theorem for pairs is strictly weaker than Ramsey's Theorem for pairs over RCA $A_{0}$.

We list here some notational conventions. We denote the set of natural numbers by $\omega$. We identify each $n \in \omega$ with the set of elements less than it, so $n=\{0,1,2, \ldots, n-1\}$. Lowercase roman letters near the beginning or middle of the alphabet $(a, b, c, i, j, k, \ldots)$ will 
denote elements of $\omega$, and lowercase roman letters near the end of the alphabet $(x, y, z, u, \ldots)$ will denote finite subsets of $\omega$. We identify a finite subset $x$ of $\omega$ of size $n$ with the $n$-tuple listing $x$ in increasing order and with the corresponding function $g: n \rightarrow \omega$. When we write $\left(x_{0}, \ldots, x_{n-1}\right) \in[\omega]^{n}$, we assume that $x_{0}<\ldots<x_{n-1}$. Uppercase roman letters near the end of the alphabet $(X, Y, Z, \ldots)$ will denote subsets of $\omega$, and uppercase roman letters near the beginning or middle of the alphabet $(A, B, C, H, I, J, \ldots)$ will denote infinite subsets of $\omega$. Given $X \subseteq \omega$, we denote the set of finite sequences of elements of $X$ by $X^{<\omega}$. We use $\sigma, \tau, \ldots$ to denote elements of $\omega^{<\omega}$. For a set $X \subseteq \omega$, we let $\operatorname{deg}(X)$ denote the Turing degree of $X$.

The topic of this paper relates to Computability Theory, Algorithmic Randomness, and Reverse mathematics. For background in Computability Theory, we recommend Soare [17] or Cooper [4]; for Algorithmic Randomness, Downey and Hirschfeldt [5], Nies [14], or Downey, Hirschfeldt, Nies and Terwijn [6]; and for Reverse mathematics, Simpson [16].

\section{Rainbows in the Arithmetical Hierarchy}

Jockusch precisely characterized the position of homogeneous sets for computable colorings in the arithmetical hierarchy.

Theorem 2.1 (Jockusch [9]).

(1) Let $n \geq 2$. There exists a computable $f:[\omega]^{n} \rightarrow 2$ such that no $\Sigma_{n}^{0}$ set is homogeneous for $f$.

(2) Suppose that $n, k \geq 2$ and that $f:[\omega]^{n} \rightarrow k$ is computable. There exists a $\Pi_{n}^{0}$ set homogeneous for $f$.

Using the above proof of the Rainbow Ramsey Theorem from Ramsey's Theorem, we easily obtain the same $\Pi_{n}^{0}$ upper bound. Indeed, we begin with the following definition. 
Definition 2.2. For each $n \in \omega$, we let $\prec_{n}$ be the reverse lexicographic ordering on $[\omega]^{n}$. That is, given $x, y \in[\omega]^{n}$ with $x \neq y$, say $x=\left(x_{0}, x_{1}, \ldots, x_{n-1}\right)$ and $y=\left(y_{0}, y_{2}, \ldots, y_{n-1}\right)$, we let $x \prec_{n} y$ if $x_{i}<y_{i}$ for the greatest $i<n$ such that $x_{i} \neq y_{i}$. Notice that, given our convention that $x_{0}<x_{1}<\ldots<x_{n_{1}}, \prec_{n}$ is a well-ordering of $[\omega]^{n}$ of order type $\omega$. Moreover, not only is $\prec_{n}$ is computable, but given $x \in[\omega]^{n}$, we can compute a canonical index for the set of elements $\prec_{n}$-below $x$.

Corollary 2.3. Suppose that $n, k \geq 2$ and that $f:[\omega]^{n} \rightarrow \omega$ is $k$-bounded. There exists a $\Pi_{n}^{0}$ set which is a rainbow for $f$.

Proof. Define $g:[\omega]^{n} \rightarrow k$ as in the proof of Theorem 1.6 using the well-ordering $\prec_{n}$. Notice that $g$ is computable. By Theorem 2.1, we may fix a $\Pi_{n}^{0}$ set $H$ homogeneous for $g$. By the proof of Theorem 1.6, we then have that $H$ is a $\Pi_{n}^{0}$ rainbow for $f$.

In fact, we get the same lower bounds for rainbows as Jockusch obtained for homogenous sets. We first handle the case when $n=2$ using an argument similar to the one given by Jockusch for Ramsey's Theorem.

Theorem 2.4. There exists a computable 2-bounded $f:[\omega]^{2} \rightarrow \omega$ such that no $\Sigma_{2}^{0}$ set is a rainbow for $f$.

Proof. It suffices to build a computable 2-bounded $f:[\omega]^{2} \rightarrow \omega$ such that no $\Delta_{2}^{0}$ set is a rainbow for $f$ because every infinite $\Sigma_{2}^{0}$ set has an infinite $\Delta_{2}^{0}$ subset, and every infinite subset of a rainbow for $f$ is also a rainbow for $f$. Using the Limit Lemma, we may fix a computable $g: \omega^{3} \rightarrow 2$ such that for every $\Delta_{2}^{0}$ set $D$, there exists an $e \in \omega$ such that

- For all $a \in D$, we have $\lim _{s} g(e, a, s)=1$.

- For all $a \notin D$, we have $\lim _{s} g(e, a, s)=0$. 
Concretely, we may let

$$
g(e, a, s)= \begin{cases}1 & \text { if } \varphi_{e, s}^{K_{s}}(a) \downarrow=1 \\ 0 & \text { otherwise }\end{cases}
$$

We now define our computable function $f$ in stages by defining $f(a, s)$ for all $a<s$ at stage $s$. Suppose we're at stage $s$. We now have $s$ substages. At substage $e<s$, suppose that there exist at least two numbers $a$ and $b$ with $a<b<s$ such that $g(e, a, s)=g(e, b, s)=1$ which have not been claimed during stage $s$ (otherwise, do nothing at this substage). Claim the least such $a$ and $b$, and define $f(a, s)=\langle a, s\rangle=f(b, s)$. Continue to the next substage. Once we've gone through all of the substages less than $s$, define $f(a, s)=\langle a, s\rangle$ for all $a<s$ which were not claimed at stage $s$, and continue to the next stage. Notice that $f$ is 2 -bounded.

Suppose now that $D$ is an infinite $\Delta_{2}^{0}$ set. Fix $e \in \omega$ such that

- For all $a \in D$, we have $\lim _{s} g(e, a, s)=1$.

- For all $a \notin D$, we have $\lim _{s} g(e, a, s)=0$.

Let $c$ be the $(2 e+2)^{\text {th }}$ element of $D$ in increasing order (recall that $D$ is infinite), and fix $t$ such that $g$ has settled on $e$ below $c$, i.e. such that

- For all $a \in D$ with $a \leq c$, and all $s \geq t$, we have $g(e, a, s)=1$.

- For all $a \notin D$ with $a \leq c$, and all $s \geq t$, we have $g(e, a, s)=0$.

Fix $s \geq \max \{c+1, t\}$ such that $s \in D$. Since the approximation to $D$ by $g$ will be correct up to $c$ at stage $s$, and since $c$ is the $(2 e+2)^{\text {th }}$ member of $D$, there will be $2 e+2$ numbers $a \leq c$ with $g(e, a, s)=1$. Since at most $2 e$ elements are claimed at stage $s$ before substage $e$, the construction at substage $e$ then claims the least unclaimed elements $a, b$ with $g(e, a, s)=$ $g(e, b, s)=1$, and defines $f(a, s)=f(b, s)$. By the minimality of $a$ and $b$ and the correctness of $g$ up to $c$, it follows that $a, b \in D$. But then $a, b, s \in D$ and $f(a, s)=f(b, s)$, so $D$ is not a rainbow for $f$. 
From here, we use a relativized version of this theorem together with the Limit Lemma to lift the result to all $n$.

Theorem 2.5. Let $n \geq 2$ and let $X \subseteq \omega$. There exists an $X$-computable 2 -bounded $f:[\omega]^{n} \rightarrow$ $\omega$ such that no $\Sigma_{n}^{0, X}$ set is a rainbow for $f$.

Proof. The proof is by induction on $n$, where the base case of $n=2$ is handled by relativizing Theorem 2.4. Suppose that $n \in \omega$ and we know the result for $n$. Fix an $X^{\prime}$-computable 2-bounded $g:[\omega]^{n} \rightarrow \omega$ such that no $\Sigma_{n}^{0, X^{\prime}}$ set is a rainbow for $g$. By the Limit Lemma, there exists an $X$-computable $g_{0}:[\omega]^{n+1} \rightarrow \omega$ such that $\lim _{s} g_{0}(x, s)=g(x)$ for all $x \in[\omega]^{n}$.

We define an $X$-computable 2-bounded $f:[\omega]^{n+1} \rightarrow \omega$ as follows. Define $f$ by letting

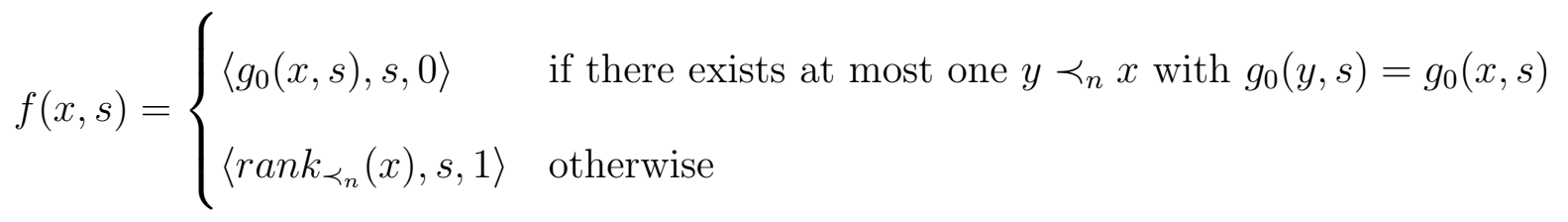

(where $\operatorname{rank}_{\prec_{n}}(x)$ is the position of $x$ in the well-ordering $\prec_{n}$ ). Notice that $f$ is $X$-computable and 2-bounded.

Suppose that $R$ is a rainbow for $f$. We claim that $R$ is a rainbow for $g$. Assume for a contradiction that $x, y \in[R]^{n}$ are such that $y \prec_{n} x$ and $g(y)=g(x)$. Fix $t \in \omega$ such that $g_{0}(z, s)=g(z)$ whenever $z \preceq_{n} x$ and $s \geq t$. Fix $s$ such that $s \in R, s \geq t$, and $s>\max (x)$. Notice that since $g$ is 2-bounded and $g_{0}(z, s)=g(z)$ for every $z \preceq_{n} x$, we have $f(z, s)=\left\langle g_{0}(z, s), s, 0\right\rangle=\langle g(z), s, 0\rangle$ for every $z \preceq_{n} x$. Hence,

$$
f(x, s)=\langle g(x), s, 0\rangle=\langle g(y), s, 0\rangle=f(y, s)
$$

contradicting the fact that $R$ is a rainbow for $f$. Therefore, $R$ is a rainbow for $g$, so $R$ is not a $\Sigma_{n}^{0, X^{\prime}}$ set. Since $\Sigma_{n}^{0, X^{\prime}}$ sets are the same as $\Sigma_{n+1}^{0, X}$ sets, it follows that $R$ is not a $\Sigma_{n+1}^{0, X}$ set. Hence, no $\Sigma_{n+1}^{0, X}$ set is a rainbow for $f$. 
In this section, we prove the following result about coloring of pairs.

Theorem 3.1. Suppose that $f:[\omega]^{2} \rightarrow \omega$ is k-bounded and computable, and that $A$ is 2random. Then there exists a rainbow $R$ for $f$ such that $R \leq_{T} A$.

3.1. Normal Colorings. In order to make some of our combinatorial bounds cleaner in the proof of Theorem 3.1, we will assume that our colorings have the following additional property.

Definition 3.2. Suppose that $f:[\omega]^{n} \rightarrow \omega$ is $k$-bounded. We say that $f$ is normal if $f(x, a) \neq$ $f(y, b)$ whenever $a \neq b$.

Proposition 3.3. Suppose that $f:[\omega]^{n} \rightarrow \omega$ is $k$-bounded and computable. There exists an infinite computable $A \subseteq \omega$ such that $f \uparrow[A]^{n}$ is normal.

Proof. We define a increasing sequence of numbers $a_{0}<a_{1}<\cdots$ recursively. We begin by letting $a_{i}=i$ for each $i<n$. Suppose that $\ell \geq n$ and we've defined $a_{i}$ for every $i<\ell$. Since $f$ is $k$-bounded, for each $c \in \omega$ there exists at most $k$ numbers $b$ such that

$$
\left\{x \in\left[\left\{a_{i}: i<\ell\right\}\right]^{n-1}: f(x, b)=c\right\} \neq \emptyset
$$

Therefore, since the set $C_{\ell}=f\left(\left[\left\{a_{i}: i<\ell\right\}\right]^{n}\right)$ is finite, there are only finitely many numbers $b$ such that

$$
\left\{x \in\left[\left\{a_{i}: i<\ell\right\}\right]^{n-1}: f(x, b) \in C_{\ell}\right\} \neq \emptyset
$$

Let $a_{\ell}$ be the least $b \in \omega$ such that

$$
\left\{x \in\left[\left\{a_{i}: i<\ell\right\}\right]^{n-1}: f(x, b) \in C_{\ell}\right\}=\emptyset
$$

We then have that $f(x, b) \notin f\left(\left[\left\{a_{i}: i<\ell\right\}\right]^{n}\right)$ for every $x \in\left[\left\{a_{i}: i<\ell\right\}\right]^{n-1}$.

Let $A=\left\{a_{i}: i \in \omega\right\}$. Notice that $A$ is computable and that $f \uparrow[A]^{n}$ is normal. 
Therefore, when considering the complexity of rainbows for computable colorings, we may assume that our colorings are normal.

3.2. Building Rainbows Directly. We now give a direct proof of the Rainbow Ramsey Theorem for pairs which does not directly appeal to Ramsey's Theorem. As in some proofs of Ramsey's Theorem, the basic idea is to build a rainbow in stages while maintaining the invariant of an infinite stock of available numbers still in play remains.

Definition 3.4. Suppose that $f:[\omega]^{2} \rightarrow \omega$ is $k$-bounded and that $F \subseteq \omega$ is finite.

- We say that $F$ is heterogeneous for $f$ if $f$ is injective on $[F]^{2}$.

- Let

$$
\operatorname{Viab}_{f}(F)=\{c \in \omega: c>F \text { and } F \cup\{c\} \text { is heterogeneous for } f\}
$$

We call $\operatorname{Viab}_{f}(F)$ the set of viable numbers for $F$ relative to the coloring $f$.

- We say that $F$ is admissible for $f$ if $F$ is heterogeneous for $f$ and $V i a b_{f}(F)$ is infinite.

The idea to build a rainbow is as follows. Suppose that we have a set $F$ which is admissible for $f$. We want to pick an element of $\operatorname{Viab}_{f}(F)$ to add to $F$ which will maintain the property of admissibility (so that we will still have infinitely much room in which to work). The following proposition says that only a small handful of elements of $V_{i a b_{f}}(F)$ would destroy this property.

Proposition 3.5. Suppose that $f:[\omega]^{2} \rightarrow \omega$ is 2-bounded and normal, and that $F \subseteq \omega$ is admissible for $f$. We then have that

$$
\mid\left\{c \in \operatorname{Viab}_{f}(F): F \cup\{c\} \text { is not admissible for } f\right\}|\leq| F \mid
$$

Proof. Let $\ell=|F|$, and suppose that $c_{0}, c_{1}, \ldots, c_{\ell} \in \operatorname{Viab}_{f}(F)$ are distinct numbers such that $F \cup\left\{c_{i}\right\}$ is not admissible for $f$ for each $i$. Since $c_{i} \in V i a b_{f}(F)$ for each $i$, we have that $F \cup\left\{c_{i}\right\}$ is heterogeneous for $f$ for each $i$. Therefore, it must be the case that $\operatorname{Viab}_{f}\left(F \cup\left\{c_{i}\right\}\right)$ is finite 
for each $i$. Fix $d \in \operatorname{Viab}_{f}(F)$ such that $d$ is greater than every element of $\operatorname{Viab}_{f}\left(F \cup\left\{c_{i}\right\}\right)$ for each $i$. For each $i$, we then have that $d \notin \operatorname{Viab}_{f}\left(F \cup\left\{c_{i}\right\}\right)$, hence $F \cup\left\{c_{i}, d\right\}$ is not heterogeneous for $f$. Since $f$ is normal, for each $i$, we may fix $a_{i} \in F$ such that $f\left(a_{i}, d\right)=$ $f\left(c_{i}, d\right)$. By the Pigeonhole Principle, there exist distinct $i, j \leq \ell$ such that $a_{i}=a_{j}$. We then have that $f\left(c_{i}, d\right)=f\left(a_{i}, d\right)=f\left(a_{j}, d\right)=f\left(c_{j}, d\right)$, contradicting the fact that $f$ is 2-bounded (because the numbers $c_{i}, c_{j}$, and $a_{i}$ are distinct).

This proposition describes a way to build a rainbow for a normal 2-bounded $f$. We begin with the set $\{0\}$, which is clearly admissible for $f$. Notice that $\operatorname{Viab}_{f}(\{0\})=\omega \backslash\{0\}$. By the Proposition, we may add one of the first two elements of $\operatorname{Viab}_{f}(\{0\})$, that is either 1 or 2 , to the set $\{0\}$ to obtain a set which is admissible for $f$. If, say, $\{0,2\}$ is admissible, then one of the first three elements of $\operatorname{Viab}_{f}(\{0,2\})$ can be added to maintain admissibility, etc.

There is nothing particular special about the case $k=2$, although the bound increases a bit for larger $k$.

Proposition 3.6. Suppose that $f:[\omega]^{2} \rightarrow \omega$ is $k$-bounded and normal, and that $F \subseteq \omega$ is admissible for $f$. We then have that

$$
\mid\left\{c \in \operatorname{Viab}_{f}(F): F \cup\{c\} \text { is not admissible for } f\right\}|\leq| F \mid \cdot(k-1)
$$

Proof. Let $\ell=|F| \cdot(k-1)$, and suppose that $c_{0}, c_{1}, \ldots, c_{\ell} \in V i a b_{f}(F)$ are distinct numbers such that $F \cup\left\{c_{i}\right\}$ is not admissible for $f$ for each $i$. Since $c_{i} \in \operatorname{Viab}_{f}(F)$ for each $i$, we have that $F \cup\left\{c_{i}\right\}$ is heterogeneous for $f$ for each $i$. Therefore, it must be the case that $\operatorname{Viab}_{f}\left(F \cup\left\{c_{i}\right\}\right)$ is finite for each $i$. Fix $d \in \operatorname{Viab}_{f}(F)$ such that $d$ is greater than every element of $\operatorname{Viab}_{f}\left(F \cup\left\{c_{i}\right\}\right)$ for each $i$. For each $i$, we then have that $d \notin \operatorname{Viab}_{f}\left(F \cup\left\{c_{i}\right\}\right)$, hence $F \cup\left\{c_{i}, d\right\}$ is not heterogeneous for $f$. Since $f$ is normal, for each $i$, we may fix $a_{i} \in F$ such that $f\left(a_{i}, d\right)=f\left(c_{i}, d\right)$. By the Pigeonhole Principle, there exists a set $Z \subseteq\{0,1, \ldots, \ell\}$ of cardinality $k$ such that $a_{i}=a_{j}$ for all $i, j \in Z$. For all $i, j \in Z$, we then have that 
$f\left(c_{i}, d\right)=f\left(a_{i}, d\right)=f\left(a_{j}, d\right)=f\left(c_{j}, d\right)$, contradicting the fact that $f$ is $k$-bounded (because the numbers $c_{i}$ for $i \in Z$, together with the common value of the $a_{i}$, are distinct).

3.3. Using Trees of Positive Measure. We outlined a direct proof of the Rainbow Ramsey Theorem for pairs in the previous section where we noted that since there were only finitely many elements of $\operatorname{Viab}_{f}(F)$ which destroy admissibility, we always had a choice which would allow us to continue. In fact, there was a computable bound on the number of elements of $\operatorname{Viab}_{f}(F)$ which would destroy admissibility. Thus, the intuition is that if we pick an element from the computable set $\operatorname{Viab}_{f}(F)$ with enough randomness, then we should never run into problems.

We make this intuition precise by building a $0^{\prime}$-computable subtree of $2^{<\omega}$ such that the set of branches has positive measure and such that any branch of the tree computes a rainbow for $f$. We first build a subtree of $\omega^{<\omega}$ from any coloring which grabs the first available number to maintain heterogeneity at each level.

Definition 3.7. Suppose that $f:[\omega]^{2} \rightarrow \omega$ is $k$-bounded and computable. We define a partial computable function $\varphi_{f}: \omega^{<\omega} \rightarrow \omega$ as follows. Let $\varphi_{f}(\lambda)=0$. Suppose that $\sigma \in \omega^{<\omega}$ and $n \in \omega$, and suppose that we have defined $\varphi_{f}(\tau)$ for all $\tau \subseteq \sigma$. Let $\varphi_{f}(\widehat{\sigma n})$ equal the $(n+1)^{\text {st }}$ element $m$ of $\omega$ greater than $\varphi_{f}(\sigma)$ such that $\left\{\varphi_{f}(\tau): \tau \subseteq \sigma\right\} \cup\{m\}$ is heterogeneous for $f$, if it exists.

Definition 3.8. Suppose that $f:[\omega]^{2} \rightarrow \omega$ is $k$-bounded.

(1) $\operatorname{Let} T_{f}=\operatorname{dom}\left(\varphi_{f}\right)$.

(2) Let $A_{f}=\left\{\sigma \in T_{f}:\left\{\varphi_{f}(\tau): \tau \subseteq \sigma\right\}\right.$ is admissible for $\left.f\right\}$.

Suppose that $f:[\omega]^{2} \rightarrow \omega$ is $k$-bounded, normal, and computable. Since $\varphi_{f}$ is a partial computable function, the tree $T_{f}$ is a c.e. subset of $\omega^{<\omega}$. Given $\sigma \in T_{f}$, we have that 
$\left\{\varphi_{f}(\tau): \tau \subseteq \sigma\right\}$ is heterogeneous for $f$ and that

$$
\operatorname{Viab}_{f}\left(\left\{\varphi_{f}(\tau): \tau \subseteq \sigma\right\}\right)=\left\{\varphi_{f}(\widehat{\sigma n}): n \in \omega\right\}
$$

Notice that $A_{f}$ is a subtree of $T_{f}$. Also, from Proposition 3.6, we know that if $\sigma \in A_{f}$, then

$$
\left|\left\{n \in \omega: \widehat{\sigma n} \notin A_{f}\right\}\right| \leq|\sigma| \cdot(k-1)
$$

The tree $T_{f}$ has the desired property that every path of $T_{f}$ corresponds to a rainbow of $f$. Indeed, if $h$ is a path in $T_{f}$ it is easy to see that $R=\left\{\varphi_{f}(\tau): \tau \subset h\right\}$ is a rainbow for $f$. Moreover, since $\varphi_{f}(\sigma)>\varphi_{f}(\tau)$ for any $\sigma \supset \tau$, it follows that $R \leq_{T} h$.

We now need to convert this subtree of $\omega^{<\omega}$ into a subtree of $2^{<\omega}$ which has positive measure many branches.

Proposition 3.9. Let $X \subseteq \omega$. Suppose that $T \subseteq \omega^{<\omega}$ is an $X$-computable tree, that $A \subseteq T$ is an arbitrary nonempty subtree of $T$ (i.e. no computability restrictions), and that $h: \omega \rightarrow \omega$ is a computable function such that for each $\sigma \in A$ we have

$$
|\{n \in \omega: \widehat{\sigma n} \notin A\}| \leq h(|\sigma|)
$$

There exists an $X$-computable tree $S \subseteq 2^{<\omega}$ such that

- $\mu\left(\left\{g \in 2^{\omega}: g\right.\right.$ is a branch of $\left.\left.S\right\}\right)>0$.

- For every branch $g$ of $S$, there exists a g-computable branch of $T$.

Proof. Define $\alpha: \omega \rightarrow \omega$ to be the computable function obtained by letting $\alpha(n)$ be the least $\ell \geq 1$ such that

$$
\frac{h(n)}{2^{\ell}} \leq \frac{1}{2^{n+2}}
$$

Define a computable function $\beta: \mathbb{N} \rightarrow \mathbb{N}$ by letting $\beta(n)=\sum_{i=0}^{n} \alpha(i)$. Define $\gamma: 2^{<\omega} \rightarrow \omega^{<\omega}$ as follows. Given $\sigma \in 2^{<\omega}$, write $\sigma$ as $\widehat{\tau_{0}} \widehat{\tau_{1}} \widehat{\ldots} \widehat{\tau_{k} \rho}$ where $\left|\tau_{i}\right|=\alpha(i)$ and $|\rho|<\alpha(k+1)$, and 
let $\gamma(\sigma)=\left(\operatorname{bin}\left(\tau_{0}\right), \operatorname{bin}\left(\tau_{1}\right), \ldots, \operatorname{bin}\left(\tau_{k}\right)\right)$ where $\operatorname{bin}(\tau)$ is the number obtained by viewing $\tau$ as written in binary. Notice that $\gamma$ is computable. Let

$$
S=\left\{\sigma \in 2^{<\omega}: \gamma(\sigma) \in T\right\}
$$

and let

$$
B=\left\{\sigma \in 2^{<\omega}: \gamma(\sigma) \in A\right\}
$$

Now $S$ is a subtree of $2^{<\omega}$ which is $T$-computable, and hence $X$-computable. Also, notice that $B$ is a subtree of $S$. By induction on $i$ it follows that

$$
\frac{\left|\left\{\sigma \in 2^{\beta(i)}: \sigma \notin B\right\}\right|}{2^{\beta(i)}} \leq \sum_{n=0}^{i} \frac{1}{2^{n+2}} .
$$

Indeed, in the case $i=0$,

$$
\begin{aligned}
\frac{\left|\left\{\sigma \in 2^{\beta(0)}: \sigma \notin B\right\}\right|}{2^{\beta(0)}} & =\frac{\left|\left\{\sigma \in 2^{\alpha(0)}: \sigma \notin B\right\}\right|}{2^{\alpha(0)}} \\
& =\frac{\left|\left\{\sigma \in 2^{\alpha(0)}: \gamma(\sigma) \notin A\right\}\right|}{2^{\alpha(0)}} \\
& \leq \frac{|\{k \in \omega: k \notin A\}|}{2^{\alpha(0)}} \\
& \leq \frac{h(|\lambda|)}{2^{\alpha(0)}} \\
& \leq \frac{1}{2^{2}}
\end{aligned}
$$


For the inductive step,

$$
\begin{aligned}
\frac{\left|\left\{\sigma \in 2^{\beta(i+1)}: \sigma \notin B\right\}\right|}{2^{\beta(i+1)}} & =\frac{\mid\left\{\sigma \in 2^{\beta(i+1)}: \sigma\lceil\beta(i) \notin B\}|+|\left\{\sigma \in 2^{\beta(i+1)}: \sigma \uparrow \beta(i) \in B \wedge \sigma \notin B \mid\right\}\right.}{2^{\beta(i+1)}} \\
& =\frac{2^{\alpha(i+1)} \cdot\left|\left\{\sigma \in 2^{\beta(i)}: \sigma \notin B\right\}\right|+\mid\left\{\sigma \in 2^{\beta(i+1)}: \sigma\lceil\beta(i) \in B \wedge \sigma \notin B \mid\}\right.}{2^{\beta(i+1)}} \\
& =\frac{2^{\alpha(i+1)} \cdot\left|\left\{\sigma \in 2^{\beta(i)}: \sigma \notin B\right\}\right|}{2^{\beta(i+1)}}+\frac{\mid\left\{\sigma \in 2^{\beta(i+1)}: \sigma\lceil\beta(i) \in B \wedge \sigma \notin B\} \mid\right.}{2^{\beta(i+1)}} \\
& =\frac{\left|\left\{\sigma \in 2^{\beta(i)}: \sigma \notin B\right\}\right|}{2^{\beta(i)}}+\frac{\left|\left\{\sigma \in 2^{\beta(i+1)}: \gamma(\sigma \uparrow \beta(i)) \in A \wedge \gamma(\sigma) \notin A\right\}\right|}{2^{\beta(i+1)}} \\
& \leq \sum_{n=0}^{i} \frac{1}{2^{n+2}}+\frac{2^{\beta(i)} \cdot h(i+1)}{2^{\beta(i+1)}} \\
& =\sum_{n=0}^{i} \frac{1}{2^{n+2}}+\frac{h(i+1)}{2^{\alpha(i+1)}} \\
& \leq \sum_{n=0}^{i} \frac{1}{2^{n+2}}+\frac{1}{2^{i+3}} \\
& =\sum_{n=0}^{i+1} \frac{1}{2^{n+2}}
\end{aligned}
$$

Since $\sum_{n=0}^{\infty} \frac{1}{2^{n+2}}=\frac{1}{2}$, it follows that $\mu\left(\left\{g \in 2^{\omega}: g\right.\right.$ is a branch of $\left.\left.S\right\}\right) \geq \frac{1}{2}$. Suppose now that $g$ is a branch of $S$. Define $f \in \omega^{\omega}$ by decoding the branch pieces in binary, i.e. letting $f=\cup_{\sigma \subset g} \gamma(\sigma)$, and notice that $f$ is a $g$-computable branch of $T$.

Theorem 3.10. Suppose that $f:[\omega]^{2} \rightarrow \omega$ is $k$-bounded and that $A$ is 2 -random. There exists a rainbow $R$ for $f$ such that $R \leq_{T} A$.

Proof. We may assume that $f$ is normal (otherwise work on an infinite computable subset such that the restriction is normal). The tree $T_{f}$ is c.e. and hence $0^{\prime}$-computable. Let $S_{f}$ be a tree obtained from Proposition 3.9. We then have that $S_{f}$ is $0^{\prime}$-computable and the set of branches of $S_{f}$ has positive measure. Relativizing a result of Kučera [11], there exists a branch $g$ of $S_{f}$ such that $g \leq_{T} A$. Let $h$ be a $g$-computable branch of $T_{f}$. Then $R=\left\{\varphi_{f}(\tau): \tau \subset h\right\}$ is a rainbow for $f$ which is $h$-computable, and hence $A$-computable. 


\section{Making Rainbows Hyperimmune}

Theorem 4.1. There exists a computable 2-bounded $f:[\omega]^{2} \rightarrow \omega$ such that every rainbow for $f$ is hyperimmune.

Proof. Recall that a set $A$ is hyperimmune iff no strictly increasing computable function majorizes $A$. That is, for $A=\left\{a_{0}<a_{1}<a_{2}<\ldots\right\}$, let $p_{A}(n)=a_{n}$, the principal function of A. A computable function $h$ majorizes $A$ if $h(x)>p_{A}(x)$ for all $x$. Thus $A$ is hyperimmune iff for all strictly increasing computable functions $h$, there exists an $x$ such that $p_{A}(x) \geq h(x)$.

Suppose first that we have one $h$ which is a total strictly increasing computable function. We will use a 2-pronged attack. The idea is to seize on two elements at a time, and ensure that if those two elements are in a particular rainbow, then there is a huge gap in that rainbow later which we ensure by making those two elements have the same color with each element of the proposed gap.

We first need to get a bound on where the first two elements that might cause us trouble will be. We consider $h(1)$, and for the sake of definiteness suppose that $h(1)=8$. Notice that if $R$ is a rainbow and $p_{R}(1) \geq 8$, then we've met this requirement trivially. The problem then is to deal with potential rainbows $R$ with $p_{R}(1)<8$, i.e. potential rainbows $R$ with two elements less than 8 . We first tackle the possibility that $0,1 \in R$. We make $(0, k)$ and $(1, k)$ have the same color for each $k$ with $8 \leq k<h(8)$. Now if $R$ is a rainbow, and $0,1 \in R$, then $k \notin R$ for all $k$ with $8 \leq k<h(8)$, hence $p_{R}(8) \geq h(8)$. We next move on to tackling the possibility that $0,2 \in R$. For definiteness, suppose that $h(8)=31$. We then make $(0, k)$ and $(2, k)$ have the same color for each $k$ with $31 \leq k<h(31)$. Now if $R$ is a rainbow, and $0,2 \in R$, then $k \notin R$ for all $k$ with $31 \leq k<h(31)$, hence $p_{R}(31) \geq h(31)$. We then in turn handle all pairs both of whose elements are less than 8. Notice that in working on this $h$, we only act on finitely many elements of $\omega$, so there is room for other computable functions to work. 
Suppose now that $\varphi_{e}$ is a partial computable function which is strictly increasing. Suppose that we are only allowed to work with numbers greater than or equal to $m$ (because other requirements have claimed the numbers less than $m)$. Suppose also that whenever $\varphi_{e}(x)[s] \downarrow$, we have $\varphi_{e}(x)[s]<s$. The problem is that we cannot just compute $\varphi_{e}(m+1)$ because it may not converge. However, we can sit around and wait for $\varphi_{e}(m+1)[s] \downarrow$, and if it never does, then we win because $\varphi_{e}$ is not total. Suppose that we see $\varphi_{e}(m+1)[s] \downarrow$, and let $n_{0}$ be a fixed stage at which this occurs. Notice that if $R$ is a rainbow (in fact any set) with $\left|\left\{x \in R: m \leq x<n_{0}\right\}\right| \leq 1$, then

$$
p_{R}(m+1) \geq n_{0}>\varphi_{e}(m+1)\left[n_{0}\right]=\varphi_{e}(m+1)
$$

We thus need to handle potential rainbows with $\left|\left\{x \in R: m \leq x<n_{0}\right\}\right| \geq 2$. We begin by handling the possibility that $m, m+1 \in R$. To do this, we make $(m, k)$ and $(m+1, k)$ have the same color for all $k \geq n_{0}$ until we see $\varphi_{e}\left(n_{0}\right)[s] \downarrow$. If this does not happen, then we win. If it does, fix such a stage $n_{1}$ at which point we can stop making $(m, k)$ and $(m+1, k)$ have the same color. Notice that if $R$ is a rainbow with $m, m+1 \in R$, then $k \notin R$ for all $k$ with $n_{0} \leq k<n_{1}$, so

$$
p_{R}\left(n_{0}\right) \geq n_{1}>\varphi_{e}\left(n_{0}\right)\left[n_{1}\right]=\varphi_{e}\left(n_{0}\right)
$$

As in the total case, we then in turn handle all pairs both of whose elements are between $m$ and $n_{0}$. Notice that in working on this $\varphi_{e}$, we only assign colors to pairs whose first component comes from a finite set, so there is room for other partial computable functions to work.

With this setup, the proof is now a finite-injury priority argument. We will build a computable 2-bounded $f:[\omega]^{2} \rightarrow \omega$ such that no computable function majorizes a rainbow for $f$. 
We let $\varphi_{0}, \varphi_{1}, \ldots$ be an enumeration of the increasing partial computable functions. In other words, we have

$$
\varphi_{e}(x) \downarrow \Rightarrow(\forall y<x)\left[\varphi_{e}(y) \downarrow<\varphi_{e}(x)\right]
$$

We will meet for all $e \in \omega$ the requirements:

$$
R_{e}: \varphi_{e} \text { total } \Longrightarrow \varphi_{e} \text { does not majorize any rainbow for } f
$$

That is, if $\varphi_{e}$ is total, then for any rainbow $R$ for $f$ we must guarantee the existence of a number $n_{R}$ such that $p_{R}\left(n_{R}\right)>\varphi_{e}\left(n_{R}\right)$.

We now outline how we would meet the single requirement $R_{0}$. We give a computable construction of $f$, where at stage $s=\langle l, k\rangle$ we define $f(l, k)$. We first define a finite (possibly empty) list of numbers $n_{0}<n_{1}<\ldots$ inductively as follows. Let $n_{0}$ be the least stage where $\varphi_{0, s}(1) \downarrow$. For $i+1 \leq \frac{\left(n_{0}\right)^{2}-n_{0}}{2}$, let $n_{i+1}$ be the least stage where $\varphi_{0, s}\left(n_{i}+1\right) \downarrow$. List the $\frac{\left(n_{0}\right)^{2}-n_{0}}{2}$ pairs $(m, l)$ with $0 \leq m<l<n_{0}$ in an order. Associate $(m, l)$ with its position in the list (a number between 1 and $\frac{\left(n_{0}\right)^{2}-n_{0}}{2}$ ).

At stage $s$, for $\langle l, k\rangle=s$, we must define $f(l, k)$. Let $i$ be maximal such that $n_{i}$ is defined at stage $s$ and $n_{i}<k$. If there exists $m<l$ such that $(m, l)=i+1$ (of course, there is at most one such), set $f(l, k)=\langle m, k\rangle$. Otherwise, set $f(l, k)=\langle l, k\rangle$.

Note that if we defined $f(l, k)=\langle m, k\rangle$, then we must have defined $f(m, k)=\langle m, k\rangle$ (since $(m, l)=i+1$ and $l \nless m$ ), so $f(l, k)=f(m, k)$. It is also (relatively) easy to see that $f$ is 2 -bounded. Finally, note that if $k>n_{i}$, then any pair $\langle l, k\rangle>k>n_{i}$, and since $n_{i}$ is defined at stage $n_{i}, n_{i}$ is defined by stage $\langle l, k\rangle$. Thus for any pair $(m, l)=i+1 \leq \frac{n_{0}^{2}-n_{0}}{2}$, for any $n_{i}<k \leq n_{i+1}, f(m, k)=f(l, k)$.

Let $R$ be any rainbow for $f$. Choose the least $m \in R$. If $m \geq n_{0}$, then $p_{R}(0)=$ $m \geq n_{0}>\varphi_{0}(0)$. If $m<n_{0}$, choose the least $l \in R$ such that $m<l$. If $l \geq n_{0}$, then $p_{R}(1)=l \geq n_{0}>\varphi_{0}(1)$. Otherwise, $m<l<n_{0}$. Let $i$ be such that $i+1=(m, l)$. Since 
$m, l \in R$, and $R$ is a rainbow for $f$, no number between $n_{i}$ and $n_{i+1}$ can be in $R$. Thus $p_{R}\left(n_{i}+1\right) \geq n_{i+1}>\varphi_{0}\left(n_{i}+1\right)$.

We now note that if $\varphi_{0}$ was not total, then it did not matter how we defined $f$. We also note that if $\varphi_{0}$ is total, we only needed to define $f$ in a particular way on finitely many pairs. Thus we can use finite injury to meet all the requirements $R_{e}$.

Stage $s$ : Let $m^{e}[s]=\max \left\{n_{i}^{q}[s]: q<e \& n_{i}^{q}[s]\right.$ is defined $\}$. Let $n_{0}^{e}[s]$ be the least stage $t \leq s$ such that $\varphi_{e, t}\left(1+m^{e}[s]\right) \downarrow$. For $i+1 \leq \frac{\left(n_{0}^{e}-m^{e}\right)^{2}-\left(n_{0}^{e}-m^{e}\right)}{2}[s]$, let $n_{i+1}^{e}$ be the least stage where $\varphi_{e, t}\left(n_{i}^{e}[s]+1\right) \downarrow$. List the $\frac{\left(n_{0}^{e}-m^{e}\right)^{2}-\left(n_{0}^{e}-m^{e}\right)}{2}[s]$ pairs $(m, l)$ with $m^{e}[s] \leq m<l<n_{0}^{e}[s]$ in an order. Associate $(m, l)$ with its position in the list.

Note that for any $q<e, n_{i}^{q}[s]<n_{0}^{e}[s]$ whenever both are defined. Note also that for any $e, n_{i}^{e}[s]<n_{i+1}^{e}[s]$ whenever both are defined. Finally, note that if $n_{i}^{e}[t]$ is defined, then the only reason it would become undefined is if some $n_{j}^{q}$ becomes defined for some $q<e$.

Now, for $\langle l, k\rangle=s$, we must define $f(l, k)$. Let $n_{i}^{e}[s]$ be maximal such that $n_{i}^{e}[s]$ is defined and $n_{i}^{e}[s]<k$. If there exists $m^{e}[s]<m<l$ such that $(m, l)=i+1[s]$ (of course, there is at most one such), set $f(l, k)=\langle m, k\rangle$. Otherwise, set $f(l, k)=\langle l, k\rangle$. Note that $f$ is 2-bounded. Indeed, assume for a contradiction that we have already defined $f(a, b)=\langle m, k\rangle$ for a third pair $(a, b)$ such that $(a, b) \neq(m, k)$. By our construction, we must have had $b=k$. Also, at the stage $t$ when $f(a, k)$ was defined, we must have had a maximal $n_{j}^{q}[t]<k<\langle a, k\rangle=t$ for some $q \geq e$. We could not have had $q=e$ and $j=i$ since then we we would have $(m, l)=(a, k)$. Sine $n_{i}^{e}[s]$ was maximal less than $k$ at stage $s$, there must have been a stage $t<t^{\prime} \leq s$ when some $n_{j^{\prime}}^{q^{\prime}}\left[t^{\prime}\right]$ with $q^{\prime}<e$ became defined. But then $k<\langle a, k\rangle=t<t^{\prime}=n_{j^{\prime}}^{q^{\prime}}\left[t^{\prime}\right] \leq m^{e}\left[t^{\prime}\right] \leq n_{0}^{e}\left[t^{\prime}\right] \leq n_{0}^{e}[s] \leq n_{i}^{e}[s]$, a contradiction.

Let $n_{0}^{e}=\lim _{s} n_{0}^{e}[s]$. Allowing "undefined" as a possibility, it is easy to see inductively that each $n_{0}^{e}$ exists. (Here, if we say $n_{0}^{e}$ is undefined, we mean the strong fact that there is a stage after which $n_{0}^{e}[s]$ is always undefined). Moreover, after the stage when $n_{0}^{e}$ has reached 
its limit, if $n_{i}^{e}$ is defined then it never changes (and there are at most $\frac{\left(n_{0}^{e}\right)^{2}-n_{0}^{e}}{2}$ such $i$, which become defined in order).

Suppose $\varphi_{e}$ is total, and let $R$ be a rainbow for $f$. Let $m=p_{R}\left(m^{e}\right)$. If $m \geq n_{0}^{e}$, then $p_{R}\left(m^{e}\right)=m \geq n_{0}^{e}>\varphi_{e}\left(m^{e}\right)$. If $m<n_{0}^{e}$, let $l=p_{R}\left(m^{e}+1\right)$. If $l \geq n_{0}^{e}$, then $p_{R}\left(m^{e}+1\right)=l \geq n_{0}^{e}>\varphi_{e}\left(m^{e}+1\right)$. Otherwise, $m^{e} \leq p_{R}\left(m^{e}\right)=m<l<n_{0}^{e}$. Let $s$ be a stage by which $n_{0}^{e}$ has reached it's limit. Let $i$ be such that $i+1=(m, l)[s]$. Note that $n_{i}^{e}=n_{i}^{e}[t]$ for any $t \geq n_{i}^{e}$. Thus for any $k>n_{i}^{e}, n_{i}^{e}[\langle l, k\rangle]<k$. Thus for any $n_{i}^{e}<k \leq n_{i+1}^{e}$, $f(l, k)=f(m, k)$. Since $m, l \in R$, and $R$ is a rainbow for $f$, no number between $n_{i}^{e}$ and $n_{i+1}^{e}$ can be in $R$. Thus $p_{R}\left(n_{i}^{e}+1\right) \geq n_{i+1}^{e}>\varphi_{0}\left(n_{i}^{e}+1\right)$.

Corollary 4.2 (Martin). Every 2-random computes a hyperimmune set. In particular,

$$
\mu\left(\left\{X \in 2^{\omega}: X \text { computes a hyperimmune set }\right\}\right)=1
$$

Proof. By Theorem 4.1, we may fix a computable 2-bounded $f:[\omega]^{2} \rightarrow \omega$ such that every rainbow for $f$ is hyperimmune. For any 2-random $A$, we know from Theorem 3.10 that $A$ computes a rainbow for $f$, and hence $A$ computes a hyperimmune set. The final statement follows using the fact that $\mu\left(\left\{A \in 2^{\omega}: A\right.\right.$ is 2 -random $\left.\}\right)=1$.

\section{Reverse Mathematics}

We refer the reader to Simpson for the basic definitions of reverse mathematics including the systems $R C A_{0}, W L_{0}$, and $A C A_{0}$.

Definition 5.1. The following definitions are made in second-order arithmetic.

(1) $\mathrm{RT}_{k}^{n}$ is the statement that every for every $f:[\mathbb{N}]^{n} \rightarrow k$, there exists a set which is homogeneous for $f$. 
(2) $\operatorname{RRT}_{k}^{n}$ is the statement that for every $k$-bounded $f:[\mathbb{N}]^{n} \rightarrow \mathbb{N}$, there exists a set which is a rainbow for $f$.

Theorem 5.2. For each fixed $n, k \in \omega$, we have that $\mathrm{RCA}_{0}+\mathrm{RT}_{k}^{n} \vdash \mathrm{RRT}_{k}^{n}$. Thus, for each fixed $n, k \in \omega$, we have that $\mathrm{ACA}_{0} \vdash \mathrm{RRT}_{k}^{n}$.

Proof. The proof of Theorem 1.6 easily goes through in $\mathrm{RCA}_{0}$. The last line follows from the fact that $\mathrm{ACA}_{0} \vdash \mathrm{RT}_{k}^{n}$ for all $n, k \in \omega$

Theorem 5.3. For each fixed $n, k \in \omega$, we have $\mathrm{RCA}_{0}+\mathrm{RRT}_{k}^{n+1} \vdash \mathrm{RRT}_{k}^{n}$.

Proof. Fix $n, k \in \omega$. Fix a model $\mathcal{M}$ of $\mathrm{RCA}_{0}+\mathrm{RRT}_{k}^{n+1}$. Suppose that $f \in \mathcal{M}$ is such that $f:[\mathbb{N}]^{n} \rightarrow \mathbb{N}$ is $k$-bounded. Define $g:[\mathbb{N}]^{n+1} \rightarrow \mathbb{N}$ by letting $g(x, i)=\langle f(x), i\rangle$ for all $x \in[\mathbb{N}]^{n}$ and all $i \in \mathbb{N}$. Notice that $g$ is $k$-bounded. Suppose that $R$ is a rainbow for g. Let $x, y \in[R]^{n}$, and fix $i \in R$ with $i>\max (x)$ and $i>\max (y)$. We then have that $g(x, i) \neq g(y, i)$, hence $f(x) \neq f(y)$. It follows that $R$ is a rainbow for $f$.

Theorem 5.4. $\mathrm{WKL}_{0} \nvdash \mathrm{RRT}_{2}^{2}$.

Proof. Fix an $\omega$-model $\mathcal{M}$ of $\mathrm{WKL}_{0}$ such that every set in $\mathcal{M}$ is low. By Theorem 2.4, we may fix a computable 2-bounded $f:[\omega]^{2} \rightarrow \omega$ such that no $\Sigma_{2}^{0}$ set is a rainbow for $f$. We then have that no low set is a rainbow for $f$, so $f \in \mathcal{M}$ but no rainbow for $f$ is in $\mathcal{M}$. It follows that $\mathcal{M} \not \mathrm{RRT}_{2}^{2}$.

Theorem 5.5. Suppose that $A$ is 2-random. There exists an $\omega$-model $\mathcal{M}$ of $\mathrm{RCA}_{0}+\mathrm{RRT}_{2}^{2}$ such that $X \leq_{T}$ A for all $X \in \mathcal{M}$.

Proof. Write $A$ as $\oplus_{n \in \omega} A_{n}$, and let

$$
\mathcal{M}=\left\{X \in 2^{\omega}: X \leq_{T} A_{0} \oplus A_{1} \oplus \cdots \oplus A_{n} \text { for some } n \in \omega\right\}
$$


Notice that $\mathcal{M}$ is a Turing ideal, so $\mathcal{M}$ is a model of $\operatorname{RCA}_{0}$. Suppose that $f:[\omega]^{2} \rightarrow \omega$ is 2-bounded and that $f \in \mathcal{M}$. Fix $n \in \omega$ such that $f \leq_{T} A_{0} \oplus A_{1} \oplus \cdots \oplus A_{n}$. By van Lambalgen's Theorem [19], we know that $A_{0} \oplus A_{1} \oplus \cdots \oplus A_{n} \oplus A_{n+1}$ is 2-random, hence by van Lambalgen's Theorem again, we know that $A_{n+1}$ is $2-\left(A_{0} \oplus A_{1} \oplus \cdots \oplus A_{n}\right)$-random. Therefore, $A_{n+1}$ is 2 - $f$-random, so there is a rainbow $R$ for $f$ such that

$$
R \leq_{T} f \oplus A_{n+1} \leq_{T} A_{0} \oplus A_{1} \oplus \cdots \oplus A_{n} \oplus A_{n+1}
$$

Thus, there exists a rainbow $R$ for $f$ such that $R \in \mathcal{M}$. It follows that $\mathcal{M}$ is a model of $\mathrm{RRT}_{2}^{2}$.

Corollary 5.6. $\mathrm{RRT}_{2}^{2}$ does not imply $\mathrm{WKL}_{0}$ over $\mathrm{RCA}_{0}$.

Proof. By Jockusch and Soare [10], we have that $\mu\left(\left\{X \in 2^{\omega}: \operatorname{deg}(X)\right.\right.$ is $\left.\left.P A\right\}\right)=0$. Thus, we may fix a 2 -random set $A$ such that $\operatorname{deg}(A)$ is not $P A$. By Theorem 5.5 , there exists an $\omega$-model $\mathcal{M}$ of $\mathrm{RCA}_{0}+\mathrm{RRT}_{2}^{2}$ such that $X \leq_{T} A$ for all $X \in \mathcal{M}$. Since $\mathcal{M}$ does not contain a set of $P A$ degree, it follows that $\mathcal{M} \not \mid \mathrm{WKL}_{0}$.

The following theorem appears in Mileti [12].

Theorem 5.7. If $A$ is hyperimmune, then $\mu\left(\left\{X \in 2^{\omega}: X\right.\right.$ computes an infinite subset of $A\})=0$.

Using this result, the following is concluded.

Corollary 5.8. There exists a computable $f:[\omega]^{2} \rightarrow 2$ such that $\mu\left(\left\{X \in 2^{\omega}: X\right.\right.$ computes a set homogeneous for $f\})=0$.

Now using this measure-theoretic distinction, we can now conclude that the Rainbow Ramsey Theorem for pairs is strictly weaker than Ramsey's Theorem for pairs over RCA $A_{0}$

Corollary 5.9. $\mathrm{RRT}_{2}^{2}$ does not imply $\mathrm{RT}_{2}^{2}$ over $\mathrm{RCA}_{0}$. 
Proof. Using Corollary 5.8, fix a computable $f:[\omega]^{2} \rightarrow 2$ such that $\mu\left(\left\{X \in 2^{\omega}: X\right.\right.$ computes a set homogeneous for $f\})=0$. Since the set of 2 -randoms has measure 1 , we may fix a 2-random set $A$ such that $A$ does not compute a set homogeneous for $f$. By Theorem 5.5, there exists an $\omega$-model $\mathcal{M}$ of $\mathrm{RCA}_{0}+\mathrm{RRT}_{2}^{2}$ such that $X \leq_{T} A$ for all $X \in \mathcal{M}$. Since $\mathcal{M}$ does not contain a set homogeneous for $f$, it follows that $\mathcal{M} \not \mathrm{RT}_{2}^{2}$.

If fact, $\mathrm{RRT}_{2}^{2}$ is sufficiently weak that it does not prove many very weak versions of Ramsey's Theorem for pairs. The following definition is from Hirschfeldt and Shore [8] and is the "stable" version of the statement that every infinite linear ordering has either an infinite ascending sequence or an infinite descending sequence.

Definition 5.10. In second-order arithmetic, SADS is the statement "For any infinite linear ordering $L$ in which each element either has finitely many predecessors or finitely many successors, there exists either an infinite ascending sequence or an infinite descending sequence in $L "$.

Theorem 5.11. $\mathrm{RRT}_{2}^{2}$ does not imply SADS over $\mathrm{RCA}_{0}$.

Proof. Fix a linear ordering $L$ of order type $\omega+\omega^{*}$ with no computable ascending or descending sequences. If $A$ is the set of elements in the $\omega$-part and $B$ is the set of elements in the $\omega^{*}$ part, then since $L$ has no computable ascending or descending sequence we certainly have that $A$ and $B$ are both immune. Moreover, both $A$ and $B$ are hyperimmune as we can argue as follows. Suppose that $\left\{D_{f(n)}\right\}_{n \in \omega}$ is a disjoint strong array such that $D_{f(n)} \cap A \neq \emptyset$ for all $n \in \omega$. Notice that for each $n \in \omega$, since $D_{f(n)} \cap A \neq \emptyset$, we have that $\min _{L}\left(D_{f(n)}\right) \in A$. Letting $C=\left\{\min _{L}\left(D_{f(n)}\right): n \in \omega\right\}$ it follows that $C$ is an infinite c.e. subset of $A$, contradicting the immunity of $A$.

Since $A$ and $B$ are both hyperimmune, we know from Theorem 5.7 that

$$
\mu\left(\left\{X \in 2^{\omega}: X \text { computes an infinite subset of either } A \text { or } B\right\}\right)=0
$$


Since every ascending sequence from $L$ computes an infinite subset of $A$, and every descending sequence of $L$ computes an infinite subset of $B$, it follows that

$$
\mu\left(\left\{X \in 2^{\omega}: X \text { computes an ascending or descending sequence from } L\right\}\right)=0
$$

Since the set of 2-randoms has measure 1, we may fix a 2-random set $A$ such that $A$ does not compute a set homogeneous for $f$. By Theorem 5.5, there exists an $\omega$-model $\mathcal{M}$ of $\mathrm{RCA}_{0}$ $+\mathrm{RRT}_{2}^{2}$ such that $X \leq_{T} A$ for all $X \in \mathcal{M}$. Since $\mathcal{M}$ does not contain an ascending or descending sequence from $L$, it follows that $\mathcal{M} \not \mid$ SADS.

Corollary 5.12. $\mathrm{RRT}_{2}^{2}$ does not imply $\mathrm{RRT}_{2}^{3}$ over $\mathrm{RCA}_{0}$.

Proof. Let $f:[\omega]^{3} \rightarrow \omega$ be a 2-bounded computable function such that no $\Delta_{3}^{0}$ set is a rainbow for $f$. Fix a 2-random set $A$ such that $A \leq_{T} 0^{\prime \prime}$. By Theorem 5.5, there exists an $\omega$-model $\mathcal{M}$ of $\mathrm{RCA}_{0}+\mathrm{RRT}_{2}^{2}$ such that $X \leq_{T} A$ for all $X \in \mathcal{M}$. Since $\mathcal{M}$ does not contain a set homogeneous for $f$, it follows that $\mathcal{M} \not \mathrm{RRT}_{2}^{3}$.

Definition 5.13. If $\vec{R}=\left\{R_{i}\right\}_{i \in \omega}$ is a sequence of sets, an infinite set $S$ is $\vec{R}$-cohesive if

$$
(\forall i)(\exists s)\left[(\forall j>s)\left(j \in S \rightarrow j \in R_{i}\right) \vee(\forall j>s)\left(j \in S \rightarrow j \notin R_{i}\right)\right] .
$$

$\mathrm{COH}$ is the statement that for every sequence $\vec{R}$, there is an $\vec{R}$-cohesive set.

Proposition 5.14. $\mathrm{COH}$ does not imply $\mathrm{RRT}_{2}^{2}$ over $\mathrm{RCA}_{0}$.

Proof. This follows from general conservation results of Hirschfeldt and Shore [8] (see their discussion after Corollary 2.21).

We close with a few questions. It is known that $\mathrm{RCA}_{0}+\mathrm{RT}_{2}^{n} \vdash \mathrm{ACA}_{0}$ for all $n \geq 3$, but the questions for the Rainbow Ramsey Theorem are open.

Question 5.15. Does there exist an $n$ such that $\mathrm{RCA}_{0}+\mathrm{RRT}_{2}^{n} \vdash \mathrm{ACA}_{0}$ ? 
Since $\mathrm{RCA}_{0}+\mathrm{RT}_{2}^{n}$ is equivalent to $\mathrm{ACA}_{0}$ for all $n \geq 3$, it follows that for any $m, n \geq 3$, $\mathrm{RT}_{2}^{m}$ is equivalent to $\mathrm{RT}_{2}^{n}$ over $\mathrm{RCA}_{0}$.

Question 5.16. Does there exist an $n \geq 3$ such that $\mathrm{RCA}_{0}+\mathrm{RRT}_{2}^{n} \vdash \mathrm{RRT}_{2}^{n+1}$ ?

\section{REFERENCES}

[1] Uri Abraham, James Cummings, and Clifford Smyth. Some results in polychromatic Ramsey theory. $J$. Symbolic Logic, 72(3):865-896, 2007.

[2] Brian Alspach, Martin Gerson, Geňa Hahn, and Pavol Hell. On sub-Ramsey numbers. Ars Combin., 22:199-206, 1986.

[3] Peter A. Cholak, Carl G. Jockusch, and Theodore A. Slaman. On the strength of Ramsey's theorem for pairs. J. Symbolic Logic, 66(1):1-55, 2001.

[4] S. Barry Cooper. Computability theory. Chapman \& Hall/CRC, Boca Raton, FL, 2004.

[5] Rod Downey and Denis R. Hirschfeldt. Algorithmic randomness and complexity. Springer-Verlag, to appear.

[6] Rod Downey, Denis R. Hirschfeldt, André Nies, and Sebastiaan A. Terwijn. Calibrating randomness. Bull. Symbolic Logic, 12(3):411-491, 2006.

[7] Pavol Hell and Juan José Montellano-Ballesteros. Polychromatic cliques. Discrete Math., 285(1-3):319$322,2004$.

[8] Denis R. Hirschfeldt and Richard A. Shore. Combinatorial principles weaker than Ramsey's theorem for pairs. J. Symbolic Logic, 72(1):171-206, 2007.

[9] Carl G. Jockusch, Jr. Ramsey's theorem and recursion theory. J. Symbolic Logic, 37:268-280, 1972.

[10] Carl G. Jockusch, Jr. and Robert I. Soare. $\Pi_{1}^{0}$ classes and degrees of theories. Trans. Amer. Math. Soc., 173:33-56, 1972.

[11] Antonín Kučera. Measure, $\Pi_{1}^{0}$-classes and complete extensions of PA. In Recursion theory week (Oberwolfach, 1984), volume 1141 of Lecture Notes in Math., pages 245-259. Springer, Berlin, 1985.

[12] Joseph R. Mileti. Partition Theorems and Computability Theory. PhD thesis, University of Illinois at Urbana-Champaign, 2004.

[13] Joseph R. Mileti. The canonical Ramsey theorem and computability theory. Trans. Amer. Math. Soc., 360(3):1309-1340 (electronic), 2008. 
[14] Andre Nies. Computability and randomness. Oxford University Press, to appear.

[15] David Seetapun and Theodore A. Slaman. On the strength of Ramsey's theorem. Notre Dame J. Formal Logic, 36(4):570-582, 1995. Special Issue: Models of arithmetic.

[16] Stephen G. Simpson. Subsystems of second order arithmetic. Perspectives in Mathematical Logic. Springer-Verlag, Berlin, 1999.

[17] Robert I. Soare. Recursively enumerable sets and degrees. Perspectives in Mathematical Logic. SpringerVerlag, Berlin, 1987. A study of computable functions and computably generated sets.

[18] Stevo Todorčević. Forcing positive partition relations. Trans. Amer. Math. Soc., 280(2):703-720, 1983.

[19] Michiel van Lambalgen. Random Sequences. PhD thesis, University of Amsterdam, 1987.

Department of Pure Mathematics, University of Waterloo, Waterloo, On, Canada N2L $3 \mathrm{G} 1$

$U R L$ : www.math. uwaterloo.ca/ csima

E-mail address: csima@math.uwaterloo.ca

Department of Mathematics, 6188 Kemeny Hall, Dartmouth College, Hanover, NH 03755

$U R L$ : www.math.dartmouth.edu/ mileti

E-mail address: csima@math.uwaterloo.ca 